\title{
ON THE TOPOLOGY OF THE EVEN-DIMENSIONAL COMPLEX QUADRICS
}

\author{
HON-FEI LAI
}

\begin{abstract}
Neat proofs are given of the explicit structures of the homotopy groups and cohomology rings of the even-dimensional complex quadrics.
\end{abstract}

1. Introduction. It is well known that the complex quadric $Q_{2 n}$, defined by the equation $z_{1}^{2}+\cdots+z_{2 n+2}^{2}=0$ in homogeneous coordinates in complex projective space $\mathbf{C} \mathbf{P}^{2 n+1}$, is diffeomorphic with the Grassmannian $\mathbf{R}_{2,2 n}$ of oriented 2-planes through the origin in Euclidean space $\mathbf{R}^{2 n+2}$. The homotopy sequence of the fibration $V_{2 n+2,2} \rightarrow \mathbf{R}_{2,2 n}$, where $V_{2 n+2,2}$ is the Stiefel manifold of orthonormal 2-frames in $\mathbf{R}^{2 n+2}$, easily yields the homotopy groups of $\mathbf{R}_{2,2 n}$ in terms of the homotopy groups of spheres, but without giving any insight into the homotopy structure of $R_{2,2 n}$. On the other hand, the explicit generators of the (integral) cohomology ring of $\mathbf{R}_{2,2 n}$ have been determined [3] using complicated calculations with Schubert varieties. In this paper, using the observation that the Hopf bundle $S^{2 n+1} \rightarrow \mathbf{C P}^{n}$ is induced from the bundle $V_{2 n+2,2} \rightarrow \mathbf{R}_{2,2 n}$, and employing standard sphere bundle and characteristic class techniques, we show that the homotopy groups and cohomology groups of $\mathbf{R}_{2,2 n}$ are isomorphic to those of $S^{2 n} \times \mathbf{C P}^{n}$, where both $S^{2 n}$ and $C P^{n}$ are naturally embedded in $R_{2,2 n}$, and give the cup product formulas for the cohomology.

2. Complex quadric and oriented Grassmannian. Let $e_{1}, \cdots, e_{2 n+2}$ be an oriented orthonormal basis of $\mathrm{R}^{2 n+2}$, and consider $\mathrm{C}^{2 n+2}$ as the complexification of $\mathbf{R}^{2 n+2}$. A natural diffeomorphism between $\mathbf{R}_{2,2 n}$ and $Q_{2 n}$ is given explicitly as follows: For any two orthonormal vectors $v_{1}, v_{2}$ in $\mathbf{R}^{2 n+2}$, the oriented 2-plane spanned by $v_{1}, v_{2}$ corresponds to $\pi\left(v_{1}+i v_{2}\right)$ in $Q_{2 n}$, where $\pi$ is the natural projection $\mathbf{C}^{2 n+2} \backslash\{0\} \rightarrow \mathbf{C} \mathbf{P}^{2 n+1}$. For details see, for example, [2, p. 280]. We orient $R_{2,2 n}$ by the complex structure on $Q_{2 n}$, and will identify the two manifolds.

Received by the editors October 30, 1973.

AMS (MOS) subject classifications (1970). Primary 57F15, 57F 20.

Key words and phrases. Complex quadric, oriented Grassmannian, Euler class, Chern classes, Poincaré dual, Gysin cohomology sequence, Hopf bundle. 
Introduce in $\mathbf{R}^{2 n+2}$ an almost complex structure $I$ by defining $J e_{2 j-1}=$ $e_{2 j}$ for $j=1, \ldots, n+1$. Then there is a natural embedding $i_{1}: \mathbf{C} \mathbf{P}^{n} \rightarrow$ $\mathbf{R}_{2,2 n}$ which sends a $J$-complex line in $\mathbf{R}^{2 n+2}$ to its underlying oriented 2 plane. Let $K$ denote its image in $\mathbf{R}_{2,2 n^{*}}$ Extend $J$ to a complex linear automorphism of $\mathrm{C}^{2 n+2}$. Take an oriented 2-plane $\omega$ spanned by orthonormal vectors $v_{1}, v_{2}$ in $\mathbf{R}^{2 n+2}$. Clearly $\omega$ is in $K$ if and only if $v_{2}=J v_{1}$, that is, if and only if $J\left(v_{1}+i v_{2}\right)=-i\left(v_{1}+i v_{2}\right)$. Following the convention in differential geometry, we can therefore say that $K$ is the $n$-dimensional projective subspace of $C P^{2 n+1}$ defined by the $(n+1)$-dimensional linear subspace of vectors of type $(0,1)$ in $C^{2 n+2}$.

There is also a natural embedding $i_{2}: s^{2 n} \rightarrow \mathbf{R}_{2,2 n}$ obtained by taking the oriented 2-planes in $\mathbf{R}^{2 n+2}$ that are spanned and oriented by $e_{1}$ and an orthogonal vector. At any $\omega \in i_{2}\left(S^{2 n}\right)$ defined by $e_{1}$ and $v$, where $v$ is in the linear space $R_{*}^{2 n+1}$ spanned by $e_{2}, \cdots, e_{2 n+2}$, the tangent space to $i_{2}\left(S^{2 n}\right)$ can be ident if ied with the $2 n$-dimensional subspace of $\mathbf{R}_{*}^{2 n+1}$ that is orthogonal to $v$. Therefore we can orient $i_{2}\left(s^{2 n}\right)$ in such a way that its tangent bundle is induced from the natural oriented $2 n$-plane bundle over $\mathbf{R}_{2,2 n}$ whose fibre over $\omega \in \mathbf{R}_{2,2 n}$ is the oriented orthogonal complement of $\omega$ in $\mathbf{R}^{2 n+2}$. With this orientation, it is straightforward to show that the intersection number of $K$ and $i_{2}\left(S^{2 n}\right)$ in $\mathbf{R}_{2,2 n}$ is equal to +1 .

We observe that there is a commutative diagram

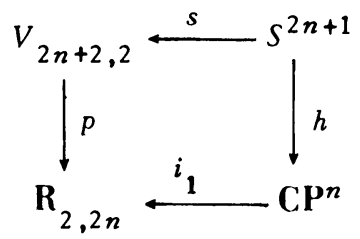

where $h$ is the Hopf map which sends a unit vector $v \in \mathbf{R}^{2 n+2}$ into the J-complex line defined by it, $p$ is the natural projection, and $s$ is the inclusion map $s(v)=(v, J v)$. The Stiefel manifold $V_{2 n+2,2}$ can also be considered as the unit tangent bundle of $s^{2 n+1}$, and $s$ is a section.

3. The homotopy groups and cohomology ring of $R_{2,2 n^{*}}$

Theorem 1. $\pi_{k}\left(\mathbf{R}_{2,2 n}\right)=i_{1 \#} \pi_{k}\left(\mathbf{C} \mathbf{P}^{n}\right) \oplus i_{2 \sharp} \pi_{k}\left(S^{2 n}\right)$ for $k \geq 1$, where \# denotes induced homomorphism.

Proof. With the notations of $\$ 2$, we can lift the inclusion $i_{2}: S^{2 n} \rightarrow$ $\mathbf{R}_{2,2 n}$ over the projection $p$ to an inclusion $i_{3}: s^{2 n} \rightarrow V_{2 n+2,2}$, by defining $i_{3}(v)=\left(e_{1}, v\right)$ for a unit vector $v$ orthogonal to $e_{1}$. We note that $i_{3}$ is the 
inclusion of $S^{2 n}$ as a fibre of the bundle $V_{2 n+2,2} \rightarrow s^{2 n+1}$, which has a section $s$. Hence

$$
\pi_{k}\left(V_{2 n+2,2}\right)=i_{3 \#} \pi_{k}\left(s^{2 n}\right) \oplus s_{\#} \pi_{k}\left(s^{2 n+1}\right) .
$$

Incorporating this into the homotopy sequences of the bundles $S^{1} \rightarrow V_{2 n+2,2}$ $\rightarrow \mathbf{R}_{2,2 n}$ and $S^{1} \rightarrow s^{2 n+1} \rightarrow \mathbf{C P} \mathbf{P}^{n}$, we get the following commutative diagram in which each three-term sequence is exact:

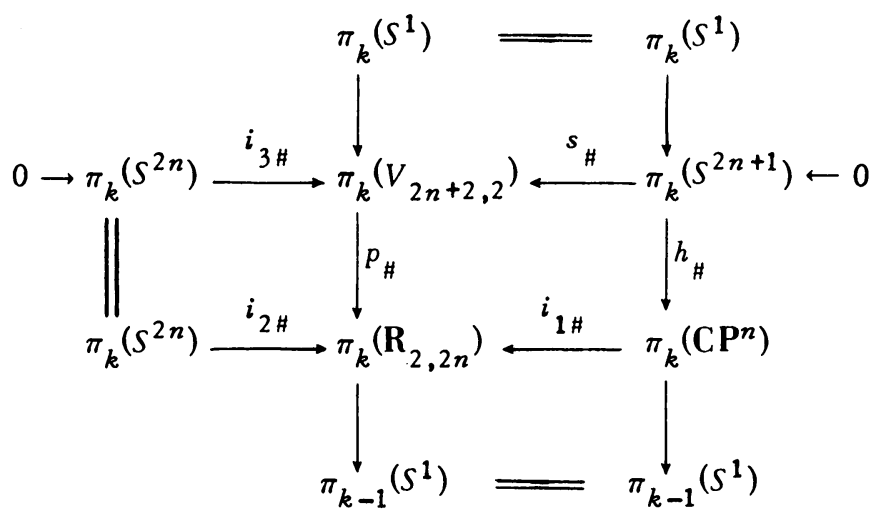

The theorem then follows easily.

There are a natural oriented $2 n$-plane bundle $\xi$ and a natural oriented 2-plane bundle $\tilde{\xi}$ over $\mathbf{R}_{2,2 n}$, where the fibre of $\tilde{\xi}$ over a point $\omega \in \mathbf{R}_{2,2 n}$ is the 2-plane $\omega$, and the corresponding fibre of $\xi$ is the orthogonal complement of $\omega$ in $\mathbf{R}^{2 n+2}$. Let $\Omega, \widetilde{\Omega}$ be their Euler classes. Let $\kappa$ be the Poincare dual of the $2 n$-dimensional homology class of $\mathbf{R}_{2,2 n}$ represented by $K$. Thus $\widetilde{\Omega} \in H^{2}\left(\mathbf{R}_{2,2 n}\right)$ and $\Omega, \kappa \in H^{2 n}\left(\mathbf{R}_{2,2 n}\right)$.

Theorem 2. The cohomology groups of $\mathbf{R}_{2,2 n}$ are isomorphic to those of $\mathbf{C P}^{n} \times S^{2 n}$, namely, for $0 \leq k \leq 4 n$,

$$
H^{k}\left(\mathbf{R}_{2,2 n}\right)= \begin{cases}\mathbf{Z} & \text { if } k \text { is even and } k \neq 2 n, \\ \mathbf{Z} \oplus \mathbf{Z} & \text { if } k=2 n, \\ 0 & \text { if } k \text { is odd. }\end{cases}
$$

As a ring, $H^{*}\left(\mathbf{R}_{2,2 n}\right)$ is generated by $\widetilde{\Omega}$ and $\kappa$, with the relation $\widetilde{\Omega}^{n+1}=$ $2 \kappa \cup \widetilde{\Omega}$. Moreover, $\Omega+\widetilde{\Omega}^{n}=2 \kappa, \kappa \cup{\widetilde{\Omega^{n}}}^{n}=(-1)^{n}, \kappa \cup \Omega=1, \kappa \cup \kappa=$ $\left(1+(-1)^{n}\right) / 2, \widetilde{\Omega}^{2 n}=2(-1)^{n}$, and $\Omega \cup \Omega=2$.

Proof. The Gys in cohomology sequence of the $2 n$-s phere bundle $V_{2 n+2,2}$ $\rightarrow s^{2 n+1}$ splits since the bundle has a section $s$. It follows easily that 


$$
H^{k}\left(V_{2 n+2,2}\right)= \begin{cases}\mathrm{Z} & \text { if } k=0,2 n, 2 n+1 \text { or } 4 n+1, \\ 0 & \text { otherwise. }\end{cases}
$$

Then the Gysin cohomology sequence for the 1 -sphere bundle $V_{2 n+2,2} \rightarrow$ $\mathbf{R}_{2,2 n}$ shows that the cohomology groups of $\mathbf{R}_{2,2 n}$ are as stated in the theorem, and that cup product with $\widetilde{\Omega}$ gives isomorphisms $H^{2 j}\left(\mathbf{R}_{2,2 n}\right) \cong$ $H^{2 j+2}\left(\mathbf{R}_{2,2 n}\right)$ for $j=0,1, \cdots, n-2, n+1, \cdots, 2 n-1$.

Since $H^{2 n}\left(\mathbf{R}_{2,2 n}\right)=\mathbf{Z} \oplus \mathbf{Z}$ and $\Omega, \widetilde{\Omega}^{n}, \kappa$ are three elements in it, there is a linear relation

$$
p \Omega+q \widetilde{\Omega}^{n}=r \kappa, \text { where } p, q, r \in \mathbf{Z} .
$$

We will calculate the values of $i_{1}^{*}$ and $i_{2}^{*}$ on the cohomology classes $\Omega, \widetilde{\Omega}$ and $\kappa$ and so solve for $p: q: r$. Let $\alpha \in H^{2}(\mathbf{C P})$ be the generator of $H^{*}\left(\mathbf{C} \mathbf{P}^{n}\right)$ which is the Poincaré dual of the homology class represented by a projective hyperplane in $\mathbf{C P} \mathbf{P}^{n}$. Then the Euler class of the Hopf bundle $\left.\left(s^{2 n+1}, h, \mathbf{C P}\right)^{n}\right)$ is $-\alpha[1]$, so $i_{1}^{*}(\widetilde{\Omega})=-\alpha$, or $i_{1}^{*}\left(\widetilde{\Omega}^{n}\right)=(-1)^{n} \alpha^{n}$. On the other hand, $i_{1}^{*}(\Omega)$ is the Euler class of the bundle $i_{1}^{*} \xi$ over $\mathbf{C P}^{n}$ which is the normal bundle to the Hopf bundle. By the duality theorem on Chern classes, $c\left(i_{1}^{*} \xi\right)=(1-\alpha)^{-1}$, and in particular, $i_{1}^{*}(\Omega)=c_{n}\left(i_{1}^{*} \xi\right)=\alpha^{n}$.

Lastly, to calculate $i_{1}^{*} \kappa$, we make use of a well-known fact, that for any embedding $j: X \rightarrow Y$ of a compact orientable manifold into another compact orientable manifold, if $D X$ denotes the cohomology class in $Y$ which is the Poincaré dual of the homology class represented by $j(X)$, and $\Omega_{\nu}$ denotes the Euler class of the oriented normal bundle of $X$ in $Y$, then $\Omega_{\nu}=j^{*}(D X)$ (see $[1, \mathrm{p} .72]$ ). We apply this fact to the embedding $i_{1}: \mathbf{C} \mathbf{P}^{n} \rightarrow Q_{2 n}$, and conclude that $i_{1}^{*}(\kappa)$ is equal to the Euler class of the normal bundle $\nu$ of $\mathrm{CP}^{n}$ in $Q_{2 n}$. We know

$$
c(\nu)=c\left(i_{1}^{*}\left(T\left(Q_{2 n}\right)\right)\right)_{c}\left(\mathbf{C} \mathbf{P}^{n}\right)^{-1}=i_{1}^{*} c\left(T\left(Q_{2 n}\right)\right)(1+\alpha)^{-(n+1)} .
$$

Using the above quoted fact on normal Euler classes again, we see that the Euler class of the normal bundle $\nu^{\prime}$ of $Q_{2 n}$ in $\mathrm{CP}^{2 n+1}$ is $j^{*}\left(D Q_{2 n}\right)=$ $j^{*}(2 \bar{\alpha})$, where $j: Q_{2 n} \rightarrow \mathbf{C} \mathbf{P}^{2 n+1}$ is the inclusion map and $\bar{\alpha}$ is defined in $\mathbf{C} \mathbf{P}^{2 n+1}$ as $\alpha$ is defined in $\mathbf{C P} \mathbf{P}^{n}$. We note that $j \circ i_{1}$ is the inclusion of $\mathbf{C P}$ in $C P^{2 n+1}$, and since by $\S 2$ this is a projective subspace in standard position, $\left(j \circ i_{1}\right)^{*} \bar{\alpha}=\alpha$. We therefore know that the total Chern class of $i_{1}^{*}\left(\nu^{\prime}\right)$ is $1+i_{1}^{*} j^{*}(2 \bar{\alpha})=1+2 \alpha$. Hence

$$
i_{1}^{*} c\left(T\left(Q_{2 n}\right)\right)=\left(j \circ i_{1}\right)^{*} c\left(T\left(\mathbf{C P}^{2 n+1}\right)\right)(1+2 \alpha)^{-1}=(1+\alpha)^{2 n+2}(1+2 \alpha)^{-1} .
$$


Finally,

$c(\nu)=(1+\alpha)^{2 n+2}(1+2 \alpha)^{-1}(1+\alpha)^{-(n+1)}=(1+\alpha)^{n+1}\left(1-2 \alpha+4 \alpha^{2}-8 \alpha^{3}+\cdots\right)$.

In particular, the Euler class of $\nu$ is

$$
\begin{aligned}
c_{n}(\nu)= & {\left[(-1)^{n} 2^{n}+\left(\begin{array}{c}
n+1 \\
1
\end{array}\right)(-1)^{n-1} 2^{n-1}+\cdots\right.} \\
& \left.+\left(\begin{array}{c}
n+1 \\
n-r
\end{array}\right)(-1)^{r} 2^{r}+\cdots+\left(\begin{array}{c}
n+1 \\
n
\end{array}\right)\right] a^{n} \\
= & -\frac{1}{2}\left[\sum_{r=0}^{n}(-1)^{r+1} 2^{r+1}\left(\begin{array}{c}
n+1 \\
n-r
\end{array}\right)\right] \alpha^{n} \\
= & -\frac{1}{2}\left[(1-2)^{n+1}-1\right] a^{n}=\sigma(n) a^{n}
\end{aligned}
$$

where

$$
\sigma(n)= \begin{cases}1, & n \text { even } \\ 0, & n \text { odd }\end{cases}
$$

Thus we have shown that $i_{1}^{*} \kappa=\sigma(n) \alpha^{n}$. This, together with the earlier formulas for $i_{1}^{*} \Omega$ and $i_{1}^{*} \widetilde{\Omega}^{n}$ and the relation (1), shows that

$$
p+(-1)^{n} q=\sigma(n) r
$$

We now calculate $i_{2}^{*}$. From $\oint_{2}$ we know that $i_{2}^{*} \xi$ is just the tangent bundle of $s^{2 n}$, so its Euler class $i_{2}^{*}(\Omega)=2 \beta$, where $\beta$ is the standard generator of $H^{2 n}\left(s^{2 n}\right)$. On the other hand, the bundle $i_{2}^{*} \xi$ has a nowhere zero section defined by $e_{1}$, so its Euler class $i_{2}^{*}(\widetilde{\Omega})$ vanishes. To evaluate $i_{2}^{*} \kappa$, we take the fundamental class $\zeta \in H_{2 n}\left(S^{2 n}\right)$ and calculate the Kronecker pairing

$$
\left.\left\langle i_{2}^{*} \kappa, \zeta\right\rangle=/ \kappa, i_{2 *} \zeta\right\rangle=\text { intersection number of } K \text { and } i_{2}\left(S^{2 n}\right)=1 \text {. }
$$

This means that $i_{2}^{*} \kappa=\beta$. Therefore from (1) we get

$$
2 p=r .
$$

The equations (2), (3) then yield $p: q: r=1: 1: 2$, so (1) reduces to

$$
\Omega+\widetilde{\Omega}^{n}=2 \kappa
$$

We now calculate the cup products. Let $\eta \in H_{4 n}\left(\mathbf{R}_{2,2 n}\right)$ be the funda- 
mental class of $\mathbf{R}_{2,2 n}$ defined by the complex structure on $Q_{2 n}$, and $\langle$, the Kronecker pairing. Then

$$
\langle\kappa \cup \Omega, \eta\rangle=\langle\Omega, \kappa \cap \eta\rangle=\left\langle\Omega, i_{1 *}\left(\mathbf{C} \mathbf{P}^{n}\right)\right\rangle=\left\langle i_{1}^{*} \Omega, \mathbf{C P}^{n}\right\rangle=1,
$$

as shown earlier. We can therefore write

$$
\kappa \cup \Omega=1 .
$$

Similarly, we prove $\kappa \cup \kappa=\sigma(n)$ and

$$
\kappa \cup \widetilde{\Omega}^{n}=(-1)^{n} .
$$

From the se formulas and the fact that $\Omega \cup \widetilde{\Omega}=0$, we can take the cup products of (4) with $\widetilde{\Omega}, \widetilde{\Omega}^{n}$ and $\Omega$, respectively, and obtain $\widetilde{\Omega}^{n+1}=2 \kappa \cup \widetilde{\Omega}, \widetilde{\Omega}^{2 n}$ $=2(-1)^{n}$ and $\Omega \cup \Omega=2$.

From (6) it follows that $\kappa \cup \widetilde{\Omega}^{n}$ is a generator of $H^{4 n}\left(\mathbf{R}_{2,2 n}\right)$, so a fortiori $\kappa \cup \widetilde{\Omega}$ is a generator of $H^{2 n+2}\left(\mathbf{R}_{2,2 n}\right)$. Also, using the above formulas for the cup products, we can calculate the determinant

$$
\left|\begin{array}{lll}
\widetilde{\Omega}^{n} \cup \widetilde{\Omega}^{n} & \widetilde{\Omega}^{n} \cup \kappa \\
\kappa \cup \widetilde{\Omega}^{n} & \kappa & \cup \kappa
\end{array}\right|=(-1)^{n},
$$

so that $\widetilde{\Omega}^{n}, \kappa$ together generate $H^{2 n}\left(\mathbf{R}_{2,2 n}\right)$. We can now write down a set of generators for the cohomology groups of $\mathbf{R}_{2,2 n},\left\{1, \widetilde{\Omega}, \widetilde{\Omega}^{2}, \ldots, \widetilde{\Omega}^{n}, \kappa, \kappa \cup \tilde{\Omega}\right.$, $\left.\cdots, \kappa \cup \widetilde{\Omega}^{n}\right\}$.

4. Some remarks. (1) In [4, Theorem 3.3], the cohomology ring structure of $\mathbf{R}_{2,2 n}$ is applied to find a lower bound for the number of parallel trangents of an embedding of a $2 n$-dimensional compact orientable manifold $M$ in $\mathbf{R}^{2 n+2}$. Now a proof without using calculations with Schubert varieties can be given by noticing that, if $d: \mathbf{R}_{2,2 n} \rightarrow \mathbf{R}_{2,2 n}$ is the map which reverses orientations of 2 -planes, we have $d^{*}(\widetilde{\Omega})=-\Omega$ and $d^{*}(\widetilde{\Omega})=-\widetilde{\Omega}$.

(2) Calculation of the normal geodesics to $K$ in $\mathbf{R}_{2,2 n}$ shows that there are two embedded $\mathbf{C P} \mathbf{P}^{n}$ in $\mathbf{R}_{2,2 n}$, denoted by $K$ and $K^{-}, 2 n$, such that $\mathbf{R}_{2,2 n} \backslash K^{-}$ is an open disc bundle over $K$. This is a more general structure than a sphere bundle over $K$ with section, and one can ask the question whether the product structure of homotopy groups holds for any space with this kind of decomposition. 


\section{REFERENCES}

1. F. Hirzebruch, Neue topologische Methoden in der algebraischen Geometrie, Ergebnisse der Mathematik und ihrer Grenzgebiete, Heft 9, Springer-Verlag, Berlin, 1956; English transl., Die Grundlehren der math. Wissenschaften, Band 131, SpringerVerlag, New York, 1966. MR 18, 509; 34 \#2573.

2. S. Kobayashi and K. Nomizu, Foundations of differential geometry. Vol. 2, Interscience Tracts in Pure and Appl. Math., no. 15, Interscience, New York, 1969. MR 38 \# 6501.

3. H.-F. Lai, Characteristic classes of real manifolds immersed in complex manifolds, Trans. Amer. Math. Soc. 172 (1972), 1-33. MR 47 \#2618.

4. - On parallel tangents of embeddings of codimension 2 in Euclidean spaces, Indiana Univ. Math. J. 22 (1973), 1171-1181.

DEPARTMENT OF MATHEMATICS, TULANE UNIVERSITY, NEW ORLEANS, LOUISIANA 70118 